\title{
An Exposition of Fractional Spurs Resulting From Nonlinear Distortion
}

\author{
Yann Donnelly, Member, IEEE, and Michael Peter Kennedy, Fellow, IEEE
}

\begin{abstract}
The interaction between quantization noise introduced by the divider controller and memoryless nonlinearities in a fractional- $N$ PLL causes fractional spurs to occur. This paper presents a comprehensive theory to explain why combinations of quantizers and memoryless nonlinearities produce fractional spurs. Necessary and sufficient conditions for spur-free behavior in the presence of an arbitrary memoryless nonlinearity or linear combinations of sets of arbitrary memoryless nonlinearities are derived. Finally, an upper limit on the number of nonlinearities for which a quantizer can exhibit spur-free performance is derived.
\end{abstract}

Index Terms-spurious, nonlinearity, phase-lock loop, PLL, quantizer, fractional spur.

\section{INTRODUCTION}

$\mathbf{F}$ RACTIONAL- $N$ PLLs are found in a variety of applications in communications systems and clocking networks [1]. Their ability to generate periodic signals with high frequency accuracy has led to their widespread use in electronic design.

The fractional- $N$ PLL is characterized by the use of a quantizer to vary the divide ratio on the feedback path of a PLL [2]. This allows fractional divide ratios to be implemented, at the expense of the introduction of phase noise. The quantizer is typically designed such that the majority of the noise that it introduces is pushed out of the pass-band of the PLL's loop response, where it can be attenuated below the level of other noise components. Digital delta-sigma modulators are commonly used.

Besides shaped phase noise, the quantizer can also contribute fractional spurs [3], [4]. So named because they become apparent when the fractional part of the divide ratio approaches a fraction with a small numerator, these are spurious frequency components which can occur at lower offset frequencies, often inside the passband of the PLL's loop response where they cannot be attenuated. Fractional spurs sometimes occur as a result of cycles that can arise during the normal operation of the quantizer. Advances in quantizer design over two decades,

Y. Donnelly was with the School of Electrical and Electronic Engineering, University College Dublin, Dublin 4, Ireland, and also with Microelectronic Circuits Centre Ireland (MCCI), based at Tyndall National Institute, Cork, Ireland. He is now with Qualcomm, Two Penrose Dock, Penrose Quay, Cork, Ireland. (e-mail: ydonnell@qti.qualcomm.com)

M. P. Kennedy is with the School of Electrical and Electronic Engineering, University College Dublin, Dublin 4, Ireland, and also with MCCI, Dublin, Ireland. (e-mail: peter.kennedy@ucd.ie)

This publication has emanated from research supported in part by a grant from Science Foundation Ireland under Grant number 13/IA/1979 and by Enterprise Ireland under Grant number TC-2015-0019. For the purpose of Open Access, the author has applied a CC BY public copyright licence to any Author Accepted Manuscript version arising from this submission many of which are summarized in a recent review [5], have eliminated these intrinsic spurs. In this work, we will assume throughout that the output of the quantizer itself is spur-free.

Other sources of fractional spurs also exist. A difficult problem is that of eliminating nonlinearity-induced fractional spurs, which occur as a result of the interaction between the quantizer-derived phase noise and inevitable nonlinearities in the PLL [3]. A range of techniques aimed at removing the quantizer-derived phase noise have been able to attenuate these spurs [6]-[11], and much progress has been made in explaining why and how spurs occur [12]-[16]. Most significantly, the possibility of spur-free performance has been proven in the case of polynomial nonlinearities [17], [18], and record low worst-case fractional spurs have been reported [19], [20].

Perrott et al. presented a linearized phase-domain model mapping the accumulated DC-free quantization noise of the quantizer to the output phase noise [21]. When this pathway includes a static nonlinearity, spurs occur [22], [23]. Galton and colleagues derived theoretical limitations on the possibility of spur-free performance of a quantizer followed by a polynomial nonlinearity of known order with unknown coefficients [24]. In this paper, we present a complementary perspective and use this to generalize to the case of a quantizer followed by an arbitrary, known memoryless nonlinearity or nonlinearities. We demonstrate how the mechanism for producing spurs can be described in purely statistical terms, and show how the spur performance can be derived from the statistical properties of the quantizer and the nonlinearity. We explicitly define conditions which result in spur-free operation for a specified nonlinearity, and illustrate how this can be used to design a quantizer which is "spur-free" after distortion. Finally, we define limitations on the realizable spur-free behavior of the quantizer in the presence of an arbitrary, known nonlinearity.

Section II demonstrates the nonlinearity-induced fractional spur, and presents non-stationary statistical concepts that are useful in explaining the causes of these spurs. In Section III, a theoretical exposition of the processes which produce these spurs is presented, and conditions which offer spur-free behavior are derived. Section IV discusses what limitations are placed on spur-free operation by these results, while Section V illustrates the results through simplified simulations. Finally, our conclusions are presented in Section VI.

\section{BACKGROUND THEORY}

\section{A. Spurious frequency components}

A simplified model of fractional spur generation in a fractional- $N$ frequency synthesizer, based on the approaches 


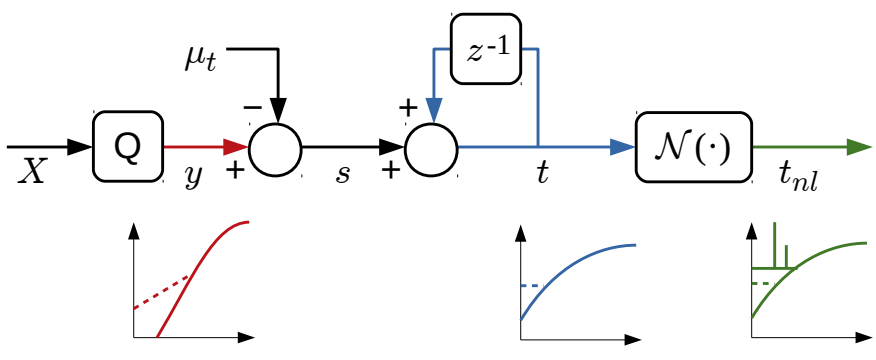

Fig. 1. Simplified model of fractional spur generation due to a nonlinearity acting on the accumulated quantization error. $X$ and $y$ denote the quantizer input and output, respectively, while $t$ and $t_{\mathrm{nl}}$ denote the accumulated quantization error before and after, respectively, application of the nonlinearity. Typical spectra are shown below for the example of a quantizer whose output, $y$, contains $2^{\text {nd }}$-order shaped quantization noise (solid curve) and $1^{\text {st }}$-order dither (dashed line). The application of the nonlinearity (denoted $N(\cdot))$ to $t$ introduces spurs into the spectrum.

of [21]-[23], is shown in Fig. 1. The quantizer Q takes a constant input $X$, and produces an output $y$ such that

$$
y[n]=\alpha+s[n],
$$

where $\alpha=\mu_{y}$ is the mean value or expectation of $y$ and $s$ is a zero-mean integer sequence. Furthermore, $-0.5 \leq \alpha \leq 0.5$ and $\alpha$ is assumed to be a multiple of a some value which we denote $1 / Q$, where $Q \in \mathbb{Z}$.

The accumulated quantization error, $t$, is a proxy for the introduction of phase noise into the system by divide ratio modulation [21]. In this paper, following the notation of [24], we define $t$ as

$$
t[n]=\sum_{k=0}^{n-1} s[k],
$$

which we can express in terms of integral and fractional components, as

$$
t[n]=m+n \alpha .
$$

A mapping function $\mathcal{N}(\cdot)$, which functions as a memoryless model of loop nonlinearities, applied to $t$ produces additional nonlinearity-induced noise terms. These can be categorized according to their manifestations in the frequency domain: (i) "linear" noise is a scaled version of the spectrum of $t$ (typically shaped to high frequencies by design), (ii) folded noise imposes a noise floor, and (iii) spurs consist of discrete frequency components. The spectrum of $t_{\mathrm{nl}}$ and the individual components are shown in the simulated spectrum of Fig. 2(c). In general, mapping through a nonlinearity introduces an elevated noise floor and spurs.

The locations of the spurs for small constant values of quantizer input, $X$, are given by [4]:

$$
f_{\text {spur, } \mathbf{k}^{\text {th }} \text { harmonic }}=\left\langle k \mu_{y} f_{\text {ref }}\right\rangle_{f}, \quad k \in \mathbb{Z},
$$

where $f_{\text {ref }}$ is the PLL reference clock frequency. $\langle\cdot\rangle_{f}$ denotes the folding operation, $\langle f\rangle_{f}=\left(f \bmod f_{\text {ref }}\right)-$ $\left(\left\lfloor 2 f / f_{\text {ref }}\right\rfloor \bmod 2\right)\left(2 f \bmod f_{\text {ref }}\right)$, where $\lfloor\cdot\rfloor$ denotes the floor function.

Fractional spurs may appear in the passband of the PLL loop response when, for example, the value of $\alpha$ approaches

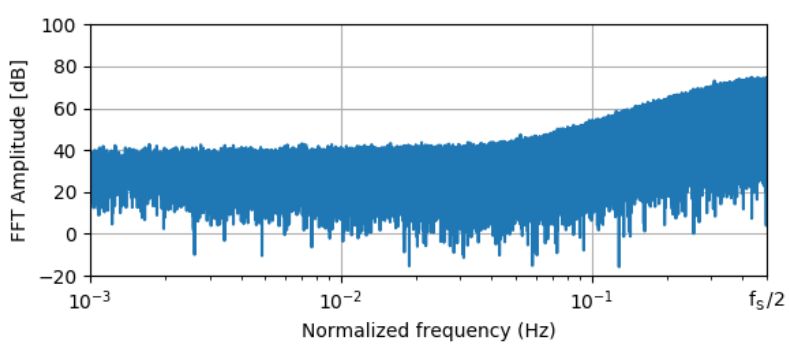

(a) $t$

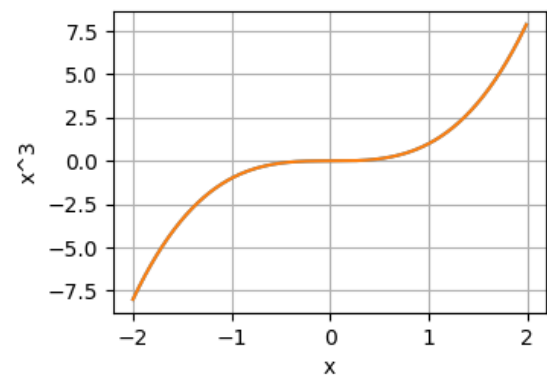

(b) $\mathcal{N}(x)$

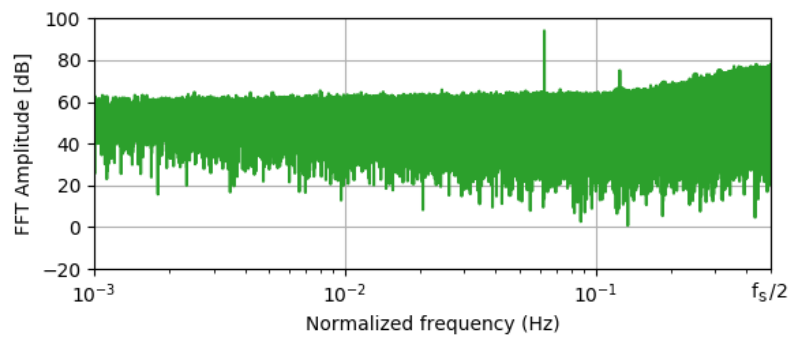

(c) $t_{\mathrm{nl}}=\mathcal{N}(t)$

Fig. 2. Example of the production of nonlinearity-derived spurs: a spur-free $t$ signal (a) interacts with an $x^{3}$ nonlinearity (b) to produce a $t_{\mathrm{nl}}$ signal with spurs (c).

1 or fractions with low-valued denominators; more general predictions for the locations of spurs can be found in [25].

While the locations of fractional spurs can be predicted, the amplitudes of the spurs are not as easy to determine. Various methods have been developed [26], [27]; one recent method consists of describing the quantization noise behavior in terms of its statistical properties [16]. We follow such a statistical approach in this work.

\section{B. Non-stationary statistics}

Because this paper relies on cyclostationary statistics to study spur formation, we will briefly introduce certain necessary terms.

A common assumption made about a stochastic system is that its output has "stationary" properties, and can be described by a single function. For example, a discrete-valued stochastic variable $x$ may be dynamic in the sense that it changes with time, but it could be described by a Probability Mass Function (PMF) which itself does not change in time. 
If a stochastic signal is non-stationary, it can be studied using ensemble techniques [28], [29] that are commonly employed in the field of thermodynamics and meteorology. Briefly, we can assign a distinct probability to a variable or property $x$ being assigned a value $a$ at sample time $n$, denoted $P_{x}(a, n)$. Note that if the PMF is stationary, then $P_{x}$ does not depend on $n$ and the solution collapses to a stationary PMF, $P_{x}(a)$.

The rejection of stationarity influences the definition of the mean. In the stationary case, there is a unique mean which is independent of $n$ and given by

$$
\mu_{x}=\sum_{a} P_{x}(a) a .
$$

In the non-stationary case, one can define a similar mean describing the expected value of $x$ in the absence of any further a priori knowledge. For this reason, we will refer to this definition as the independent mean to emphasize that it does not depend on any additional factors.

In the non-stationary case, however, the behavior of $x$ does depend on time. With this knowledge in hand, one can also define the ensemble mean, namely the expected value of $x$ at a given moment in time, $n$ [28], [29]:

$$
\mu_{x}[n]=\sum_{a} P_{x}(a, n) a .
$$

In this paper, we will deal with processes that are nonstationary, but whose time-varying stochastic properties are assumed to vary in a periodic manner. Thus, for some time period $Q$ these cyclostationary processes obey the constraint $P_{x}(a, n)=P_{x}(a, k Q+n)$ for all $k \in \mathbb{Z}$. In this case, both mean definitions are related through:

$$
\mu_{x}=\frac{1}{Q} \sum_{n=0}^{Q-1} \mu_{x}[n] .
$$

Fig. 3 offers a graphical illustration of a cyclostationary process and how the properties of the system related to each mean. The statistical properties of $x$, exemplified by the PMF, vary in time but repeat with period $N=4$. The ensemble mean, $\mu_{x}[n]$, is obtained by averaging across all possible states at a given instant in time, while the independent mean, $\mu_{x}$, is obtained by averaging across all possible states and instances in time. It follows that $\mu_{x}[n]$ is a time-varying sequence, while $\mu_{x}$ is a scalar value.

\section{The spur-free condition for polynomial nonlinearities}

The identification of conditions ensuring spur-free behavior is rarely undertaken in the literature. Familier and Galton derived necessary and sufficient conditions to guarantee spurfree behavior of $t_{\mathrm{nl}}$ when a nonlinearity $\mathcal{N}(\cdot)$, which can be represented by a finite-order polynomial, is applied to the accumulated quantizer error, $t$ [24]. This paper builds upon the key findings of that paper which we will recall here for context.

The findings of [24] relate to spurs defined as frequency components for which the periodogram is unbounded. The

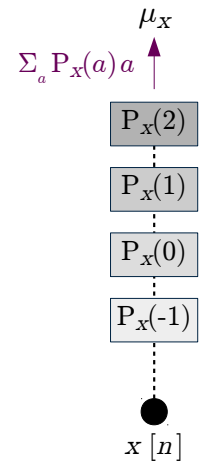

(a)

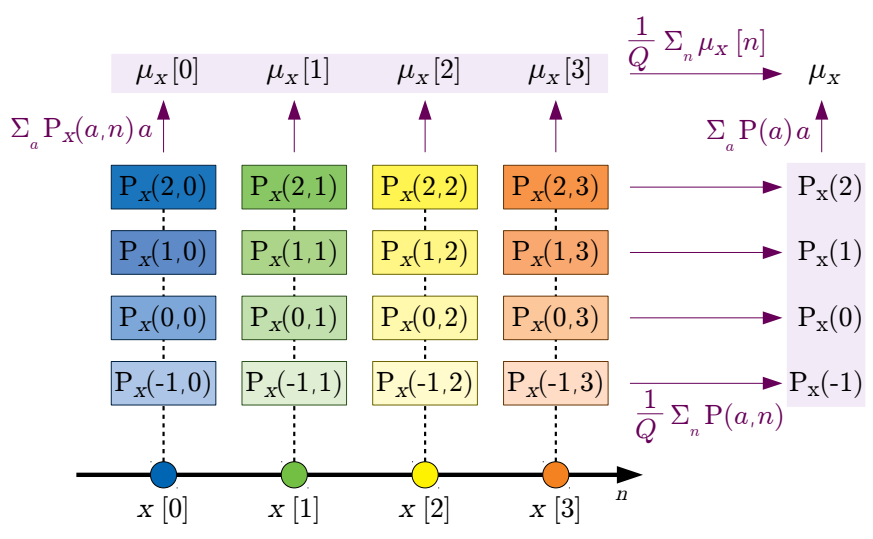

(b)

Fig. 3. Illustration of a random variable with stationary PMF (a) and a random variable with cyclostationary PMF (b), where $Q=4$ such that $P_{x}(a, 0)=$ $P_{x}(a, 4)$, etc.. Graphically depicted are the state-time probabilities (centre), ensemble mean (top) and independent mean (top right).

paper identifies a key property $\beta_{R}^{(p)}[n][24$, Eq. (28)], defined by $^{1}$

$$
\beta_{R}^{(p)}[n]=\sum_{m} P_{R}[m, n](m-\langle n \alpha\rangle)^{p},
$$

where the function $P_{R}[m, n]$ quantifies the relative frequency of $\lceil t[k Q+n]\rceil=m$ for a given spur period $Q$ over the time range $0 \leq n<R Q$, and $\langle x\rangle \equiv x \bmod 1$.

It is proven that spurs, as defined above, will not occur when $t$ encounters a polynomial function of order $p$ or less if the limit of $\lim _{R \rightarrow \infty} \beta_{R}^{(p)}[n]$ is independent of $n$. In other words, a quantizer is guaranteed to offer spur-free performance in the presence of polynomial nonlinearities of $p^{\text {th }}$-order or less if $\lim _{R \rightarrow \infty} \beta_{R}^{(p)}[n]$ is constant.

\section{NONLINEARITIES AND SPURS: THE STATISTICAL LINK}

Quantizer design normally focuses primarily on spectral shaping of the quantization noise. This section examines the phenomenon of nonlinearity-derived fractional spurs, and demonstrates how a statistical description of the phase noise

\footnotetext{
${ }^{1}$ The original definition of [24] replaces $n \alpha$, shown here, with $(n+1) \alpha$, consistant with a definition of $t[n]=\sum_{k=0}^{n} s[k]$, as opposed to the definition given in Eq. (2). Both definitions are functionally equivalent and the change being made solely to aid clarity.
} 
offers a useful and intuitive means of explaining the production of these spurs when the nonlinearity is driven by a spurfree source. This approach offers a complimentary perspective to and generalizes the findings of [24]. We prove that the amplitudes of the fractional spurs produced by an arbitrary nonlinearity can be minimized through careful codesign of the quantizer's spectral and statistical properties.

\section{A. Why do spurs "appear" in the presence of a nonlinearity?}

One of the difficulties in gaining insight into the origins of fractional spurs lies in the apparent paradox that the spur free quantization noise signal, $t$, when presented with even very simple nonlinearities consisting of nothing more than static mapping functions, can produce a noise signal $t_{\mathrm{nl}}$ that contains prominent spurs [30]. For example, Fig. 2 illustrates how, even if the spectrum of the $t$ signal is spur-free, mapping it through a nonlinear function as simple as $\mathcal{N}: x \mapsto x^{3}$ is sufficient to produce spurs in the spectrum of $t_{\mathrm{nl}}$.

Additional insight into fractional spurs can be obtained by describing the quantization noise signal using its statistical properties. As part of this approach, we focus on the cyclostationary statistical properties of both $t$ and $t_{\mathrm{nl}}$. Both the linear and folded noise components are typically aperiodic and "noise-like", so the statistical properties of these components should not exhibit any periodic dependence on time. Therefore, the cyclostationary behavior of the signal can be attributed to the remaining fractional spur component.

While we will show that attributing the spur to time-varying statistical properties is an effective method of describing and quantifying fractional spurs, justification is needed for why the statistical properties of $t_{\mathrm{nl}}$ would not be stationary; and even then, why they would vary in a periodic manner, as aperiodic changes in these properties would not produce spurs. Furthermore, we still haven't properly addressed the question of why, even if spurs are absent from $y$ and $t$, they may appear in $t_{\mathrm{nl}}$ after encountering the loop nonlinearity.

In discussing this problem, we will first use the example of a MASH quantizer, which is a simple but effective digital quantizer that is widely used to implement fractional division in frequency synthesizers. We previously mentioned the large body of prior work concerned with describing and eliminating the periodic (limit cycle) behavior of the MASH quantizer; we will now briefly summarize it as it relates to reducing fractional spurs. This digital quantizer can be considered to be a Finite State Machine (FSM) with a bounded number of states. In its simplest form, this means that, when its input is constant, its output must be periodic, and strong spurs in the quantizer output will occur for certain values of $X$.

Two principal approaches have been employed to overcome the problem of short cycles. The first approach leads to "maximum sequence length" techniques which guarantee a large output period; in effect, this allows spurs to occur, but ensures that they are sufficiently numerous (for large quantizers, which allow very large output periods) that the noise power of each individual spur is minimal [31], [32]. The second approach consists of applying dither to the quantizer in a manner that does not too strongly degrade the frequency spectrum of the quantizer output, but breaks up the periodicity of the trajectories [1], [33], [34]. When used correctly, both approaches succeed in removing spurs from the quantizer output, $y$, and accumulated output, $t$, but not from $t_{\mathrm{nl}}{ }^{2}$ Alternative quantizers have also been described whose unmodified output and accumulated output are inherently spur-free [9]. Hence, the cause of spurs in $t_{\mathrm{nl}}$ must not lie with the choice of FSM implementation.

We shall, therefore, ignore the particular choice of quantizer and represent it as a black box in the remainder of the paper. We assume that its output, $y$, is constrained in such a way that $t$ remains bounded. We will also treat $y$, and other signals derived from it, using a statistical approach. This does not imply that these signals are necessarily stochastic in nature, as the quantizer may well be a completely deterministic system. Rather, this approach is taken because statistical methods offer an intuitive way to describe how spurious components arise out of interactions between these signals and loop nonidealities.

In the frequency synthesizer application, the quantizer's purpose is to produce, from an $m$-bit input $X$, a discretetime signal, $y$, which is (i) integer-valued and (ii) has a mean of $\alpha$. Note that the former naturally arises from the digital implementation of the quantizer, while the latter is a statement of the quantizer's purpose and must be true if the quantizer is functioning correctly. We will explicitly define these properties in order to highlight them:

$$
\begin{aligned}
& \text { Property 1a: } y[n] \in \mathbb{Z} \text {, and } \\
& \text { Property 2a: } \mu_{y}=\alpha .
\end{aligned}
$$

Let us define the running sum of this output, $y_{\text {sum }}[n]=$ $\sum_{k=0}^{n-1} y[k]$, as an output of the black box. The above properties translate into the following corresponding pair of properties:

$$
\begin{aligned}
& \text { Property 1b: } y_{\text {sum }}[n] \in \mathbb{Z} \text {, and } \\
& \text { Property 2b: } \mu_{y_{\text {sum }}}[n]=n \alpha .
\end{aligned}
$$

Property $1 \mathrm{~b}$ follows from the observation that the sum of integers is itself an integer, while Property $2 b$ follows from the distributive property of the mean. Note that we have chosen to show the ensemble mean, defined previously, which will allow us to follow the evolution of $y_{\text {sum }}$ and its differences over time.

Since $-1 / 2 \leq \alpha<1 / 2$, it follows that, in general, $n \alpha \notin \mathbb{Z}$, despite Property $1 \mathrm{~b}$ stating that $y_{\text {sum }} \in \mathbb{Z}$. This is to be expected, as the purpose of the quantizer is to produce a fractional output mean.

The properties also tell us another important thing about this accumulation signal. Property $1 \mathrm{~b}$ defines the range of $y_{\text {sum, }}$, in this context taken to mean the set which includes all potential

\footnotetext{
${ }^{2}$ It is sometimes suggested that the addition of dither directly breaks up periodicities in a signal, thus removing spurs, and therefore that it follows that the application of dither would also remove spurs from $t_{\mathrm{nl}}$. This logic appears to follow from the case of intrinsic spurs in $t$ mentioned above, where applying dither results in increased utilisation of FSM states, breaking up the limit cycles which are responsible for these spurs.

However, we will shortly show that the nonlinearity-derived spurs are a completely unrelated phenomenon. The application of dither doesn't inhibit the mechanism producing these spurs, although "zeroth order" dither with a non-zero mean may modify the effective quantizer output mean, thus slightly modifying the value of $f_{\text {spur }}$.
} 
values of $y_{\text {sum }}$ which have a non-zero probability of occurring, and states that this range is time-invariant; while Property $2 b$ defines the expected value of $y_{\text {sum }}$, and states that this property is time-varying. $y_{\text {sum }}$ therefore has a time-varying statistical property, namely the expected value of the output.

The expected value of $y_{\text {sum }}$ may be time-varying, but it is not periodic. Let us denote another output of the black box as $t$, which we previously defined and which relates to $y_{\text {sum }}$ through $t[n]=y_{\text {sum }}[n]-n \mu_{y}$. This signal is the DC-free quantization noise mentioned earlier. This results in the following pair of properties:

$$
\begin{aligned}
& \text { Property 1c: }(t[n]+n \alpha) \in \mathbb{Z} \text {, and } \\
& \text { Property 2c: } \mu_{t}[n]=0 .
\end{aligned}
$$

We now have a qualitatively different signal, in the sense that its expected output is time-invariant and its range is timevarying for non-integer values of $\alpha$. However, it still has a time-varying statistical property, namely the range of $t$.

By definition, $\mathbb{Z}+\mathbb{Z}=\mathbb{Z}$, so Property $1 \mathrm{c}$ can be rewritten as $t[n]+\langle n \alpha\rangle \in \mathbb{Z}$. Since $\mathbb{Z}$ is independent of $n$, this property is periodic if $\langle t[n]\rangle=\langle n \alpha\rangle$ is periodic.

Note that since $\alpha$ is a multiple of some $1 / Q$, then:

$$
\begin{aligned}
\langle t[n+Q]\rangle & =\langle(n+Q) \alpha\rangle \\
& =\langle n \alpha+Q \alpha\rangle \\
& =\langle n \alpha\rangle \\
& =\langle t[n]\rangle .
\end{aligned}
$$

Hence, the range property is periodic in $n$ with a period of $Q$.

The key question is how spurs can be absent from $t$, but reappear in $t_{\mathrm{nl}}$, when both signals are related through a simple nonlinear mapping. The condition for spur-free operation presented in [24], and repeated here as Eq. (8), highlights the ability of the ensemble mean to deliver insight into the spurious behavior of a signal. Applying Property 1c to Eq. (6), we obtain the following expression for the ensemble mean of $t$ :

$$
\mu_{t}[n]=\sum_{m} P_{t}(m-\langle n \alpha\rangle, n)(m-\langle n \alpha\rangle) .
$$

If we observe that $P_{t}(a, n)=\lim _{R \rightarrow \infty} P_{R}(a, n)$ as defined in [24], then from comparison with Eq. (8) it becomes clear that $\mu_{t}[n]=\lim _{R \rightarrow \infty} \beta_{R}^{(0)}[n]$. It follows that the analysis of [24] proves that $t$ is spur-free if its ensemble mean $\mu_{t}[n]$ is independent of $n$, i.e. remains constant in time.

Similarly, $\mu_{t^{p}}[n]=\lim _{R \rightarrow \infty} \beta_{R}^{(p)}[n]$; hence, Eq. (8) also establishes that $t^{p}$ is spur-free if its ensemble mean $\mu_{t^{p}}[n]$ remains constant. From this observation, one can extend the approach of [24] and prove that, after applying an arbitrary static nonlinearity mapping $\mathcal{N}(\cdot)$ to $t$, the resultant $t_{\mathrm{nl}}=\mathcal{N}(t)$ is spur-free if its ensemble mean, defined by

$$
\mu_{t_{\mathrm{nl}}}[n]=\sum_{m} P_{t}(m-\langle n \alpha\rangle, n) \mathcal{N}(m-\langle n \alpha\rangle),
$$

is constant. Note that the ensemble mean in all cases depends on the probabilities $P_{t}(m-\langle n \alpha\rangle, n)$, which depend in turn on the quantizer design.

This observation offers an intuitive means of explaining the ability of nonlinear mappings to induce spurious frequency

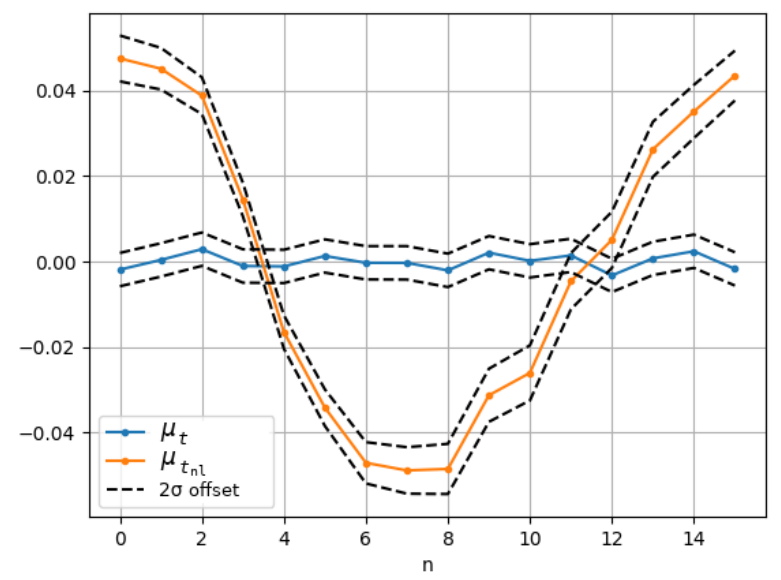

Fig. 4. Illustration of the change in the simulated ensemble mean after application of the $x^{3}$ nonlinearity, as illustrated in Fig. 2. $t$ is spur-free because the ensemble mean is constant (with some deviation seen, resulting from the finite simulation length). Application of the nonlinearity produces a signal, $t_{\mathrm{nl}}$, whose ensemble mean is a periodic function, that produces the spurs in Fig. 2(c).

components in spur-free signals. In our previous example of a simple MASH quantizer, spurs are present because, while the correctly designed quantizer ensures that the independent mean is a constant $\mu_{y}=\alpha$, the ensemble mean of the output, $\mu_{y}[n]$, is time-varying and periodic. The spur-free quantizer, on which our black box is based, has a constant expectation $\mu_{y}[n]$, and hence a spur-free output. $\mu_{y_{\text {sum }}}[n]$ is time-varying, but not periodic (Property $2 \mathrm{~b}$ ), while the range of $t$ is periodic in time (Property 1c), but $\mu_{t}[n]$ is fixed (Property 2c), so $t$ is still spur-free.

If, however, we apply a nonlinear mapping function $\mathcal{N}$ to $t$, then this has a $\mu_{t_{\mathrm{nl}}}[n]$ that does not necessarily remain constant in time, since

$$
\sum_{a} P_{t}(a, n) a=\sum_{a} P_{t}(a, n) \mathcal{N}(a)+C
$$

where $a=m-\langle n \alpha\rangle$ and $C$ is some constant, is not true in general.

To return to the example given at the start of the section, shown in Fig. 2, we can attribute the absence of fractional spurs in Fig. 2(a) but the presence of fractional spurs in Fig. 2(c) to $\mu_{t}[n]$ being constant and $\mu_{t_{\mathrm{nl}}}[n]$ time-varying. Plots of ensemble means for the simulated $t$ and $t_{\mathrm{nl}}$ signals of Fig. 2 are shown in Fig. 4, demonstrating that changes in the ensemble mean offer an explanation for the appearance of fractional spurs after application of the nonlinearity.

\section{B. Conditions which result in spur-free behavior}

We have already established that spurs are present in the spectrum of $y_{\mathrm{nl}}$ if its ensemble mean changes with time. We can now build upon this observation to determine what conditions result in spur-free behavior of $t_{\mathrm{nl}}$.

As our use of the phrase "nonlinear" indicates, it is known that spurs are present in the mapped output, $t_{\mathrm{nl}}$, when the mapping function contains a nonlinearity, for example polynomial 
terms of order greater than 1. Mappings which result only in an increase in amplitude (gain) or a fixed offset, or both, do not produce spurs when $t$ is spur-free to begin with.

We can demonstrate this using the general affine mapping function, described by $\mathcal{N}: x \mapsto G x+o$, where $G$ is a gain factor and $o$ is a constant offset. If the quantizer has been defined in such a way that $t$ is spur-free-ie. that $\mu_{t}[n]=C$ then this affine transform will also result in spur-free operation:

$$
\begin{aligned}
\mu_{t_{\mathrm{nl}}}[n] & =\sum_{m} P_{t}(m-\langle n \alpha\rangle, n) \mathcal{N}(m-\langle n \alpha\rangle) \\
& =\sum_{m} P_{t}(m-\langle n \alpha\rangle, n)(G(m-\langle n \alpha\rangle)+o) \\
& =G \sum_{m} P_{t}(m-\langle n \alpha\rangle, n)+o \sum_{m} P_{t} \\
& =G \mu_{t}[n]+o \\
& =G C+o,
\end{aligned}
$$

where $t_{n l}=\mathcal{N}(t)$, as before. We have used the fact that the summation of the PMF at a given moment in time, $\sum_{a} P_{t}(a, n)$, is 1 by definition, and have applied the definition of $\mu_{t}[n]$ given by Eq. (11).

For a mapping other than a simple linear or affine one, it is highly improbable that both $t$ and $t_{\mathrm{nl}}$ could both have a constant ensemble mean and, hence, both be spur-free. To underline the statement in the previous section regarding the improbability of $t$ remaining spur-free after an arbitrary nonlinear mapping, consider the "square" mapping function $\mathcal{N}: x \mapsto x^{2}$ as a slightly more complex example. In this case,

$$
\begin{aligned}
\mu_{t_{\mathrm{nl}}} & =\sum_{m} P_{t}(m-\langle n \alpha\rangle, n) \mathcal{N}(m-\langle n \alpha\rangle) \\
& =\sum_{m} P_{t}(m-\langle n \alpha\rangle, n)(m-\langle n \alpha\rangle)^{2} \\
& =\sum_{m} P_{t}(m-\langle n \alpha\rangle, n)\left(m^{2}-2 m\langle n \alpha\rangle+\langle n \alpha\rangle^{2}\right) .
\end{aligned}
$$

Since we are assuming that $\mu_{t}[n]=C$, some constant, then, $\sum_{m} P_{t}(m-\langle n \alpha\rangle, n) m=\sum_{m} P_{t}(m-\langle n \alpha\rangle, n)\langle n \alpha\rangle+C$,

which can be substituted into the middle term to produce:

$$
\begin{aligned}
\mu_{t_{\mathrm{nl}}}[n] & =\sum_{m} P_{t}(m-\langle n \alpha\rangle, n)\left(m^{2}-2 m\langle n \alpha\rangle+\langle n \alpha\rangle^{2}\right) \\
& +2 C\langle n \alpha\rangle \\
& =\sum_{m} P_{t}(m-\langle n \alpha\rangle, n)\left(m^{2}-\langle n \alpha\rangle^{2}\right)+2 C\langle n \alpha\rangle \\
& =\sum_{m} P_{t}(m-\langle n \alpha\rangle, n) m^{2}+2 C\langle n \alpha\rangle-\langle n \alpha\rangle^{2} .
\end{aligned}
$$

Therefore, $\mu_{t_{\mathrm{nl}}}[n]$ will depend explicitly on $n$ for all but the most carefully tailored probabilities $P_{t}$.

By extension, the condition that the ensemble mean should remain constant with $n$ for $t_{\mathrm{nl}}$ to be spur-free amounts to defining a collection of up to $Q$ simultaneous equations which must be satisfied. These conditions cannot be satisfied, for an arbitrary nonlinearity, by a simple quantizer such as a MASH.
While the condition $E\left(y_{\mathrm{nl}}[n]\right)=$ constant for all $n$ is difficult to satisfy in general, quantizers have been designed to provide spur-free operation in the case of a subset of nonlinearities, such as the $\mathcal{N}: x \mapsto x^{2}$ case described here [17], [18]. Spur-free quantizers do not currently exist in the literature for more complex nonlinearities; however, the above findings strongly suggest the possibility of a more general solution to guaranteeing spur-free behavior.

Furthermore, it is clear that a hypothetical "nonlinearityproof" quantizer, promising spur-free behavior for any a priori unknown nonlinearity, is impossible. This is because, for any nonlinearity mapping function $\mathcal{N}$, there is an accompanying spur-free condition that places different constraints on the quantizer probabilities $P_{k}[n]$ for different nonlinearities. Hence, the conditions for spur-free behavior cannot be met for every possible nonlinearity. This provides another perspective and is consistent with the original result of [24], which proves that, for a quantizer with a given amplitude of $t$, there is an upper bound on the order of the polynomial nonlinearity for which it can guarantee spur-free operation. In Section IV, we will extend this finding to define an upper bound on the spurfree performance for a collection of arbitrary nonlinearities.

\section{LIMITATIONS ON SPUR-FREE OPERATION}

We will complete our theoretical analysis of the causes of nonlinearity-derived fractional spurs with some observations on what limitations are placed on the quantizer if spur-free operation is required.

We have previously presented the constraints introduced in [24] which guarantee the spur-free behavior of $t$ after the application of a finite-order polynomial function, and discussed how these constraints intuitively relate to the ensemble mean of $t$ and extend to the more general case of an arbitrary static nonlinearity. We have also explained how these findings appear to disallow the possibility of a generic "nonlinearityproof" quantizer that would offer spur-free performance for any nonlinearity function. [24] reaches a similar conclusion in the specific case of polynomial nonlinearities. The constraint determined in the latter paper can be formulated as follows:

Theorem FG1: Consider a quantizer designed such that $t$ has an amplitude of $T$, and a set of nonlinear functions of the form $\mathcal{N}: x \mapsto x^{p}$ where $1 \leq p \leq \Gamma$. Then $t_{\mathrm{nl}}$ must contain spurs for at least one of these nonlinearities if $\Gamma>T-1$.

This theorem places a constraint on the highest order that can be made spur-free, for a given amplitude of $t$. We can define an analogous constraint, but one that applies for any set of arbitrarily defined nonlinearities.

Firstly, define a nonlinear function as "non-trivial" if it does not take the form $\mathcal{N}: x \mapsto C$, where $C$ is a constant. Note that the identity function, $\mathcal{N}: x \mapsto x$, although defining the linear case, is not considered trivial as a quantizer could offer spurfree operation for a number of nonlinearities without being 
spur-free in the linear case. ${ }^{3}$

Secondly, define two nonlinear functions $\mathcal{N}_{a}, \mathcal{N}_{b}$ as "affinerelated" if one can be derived from the other by an affine transformation-ie. $\mathcal{N}_{a}: x \mapsto f(x)$ and $\mathcal{N}_{b}: x \mapsto$ $G f(x)+o$, for some constant $G, o \in \mathbb{R}$-in which case spur-free operation for one implies spur-free operation for the other, as demonstrated in Section III-B. Similarly, define a collection of nonlinear functions $\mathcal{N}_{a}, \mathcal{N}_{b}, \ldots$ as "affinerelated" if any one nonlinear function in the set can be defined by linear combinations of the others or affine transformations thereof. Therefore, affine-related functions have identical spurfree conditions which should not be considered as separate constraints.

Then,

Theorem DK1: Consider a quantizer designed such that $t$ has an amplitude of $T$, and a set of $\Gamma$ arbitrary non-trivial and affine-unrelated nonlinear functions. Then $t_{\mathrm{nl}}$ must contain spurs for at least one of these nonlinearities if $\Gamma>T-1$.

Proof. $\mu_{t}[n]$ must remain constant for spur-free operation in the presence of a nonlinearity. This problem can be expressed as a set of simultaneous equations.

At each moment in time, $n$, the right-hand side of Eq. (11) contains $T$ variables associated with each $m$ term in $P_{t}(m-$ $\langle n \alpha\rangle, n)$; these reduce to $(T-1)$ variables after the application of the fundamental property $\sum_{m} P_{t}(m-\langle n \alpha\rangle, n)=1 . \mu_{t}[n]$ is periodic with period $M$, so the equality must hold over $M$ values of $n$, resulting in $(T-1) M$ variables associated with the $P_{t}(m-\langle n \alpha\rangle, n)$ terms. Note that we are interested in specifying an upper bound, so we can set aside, for the moment, the requirement that the $P_{t}(m-\langle n \alpha\rangle, n)$ terms must be positive.

The left-hand side of Eq. (11) must remain constant for each choice of nonlinearity. If we have $\Gamma$ nonlinearities, this results in $\Gamma$ additional variables associated with these constants.

In total, we now have a set of $\Gamma M$ simultaneous equations, with $(T-1) M+\Gamma$ unknowns. A solution exists if

$$
\begin{aligned}
\Gamma M & \leq(T-1) M+\Gamma \\
\Gamma & \leq \frac{(T-1) M}{M-1} \\
\Gamma & \leq(T-1)+\frac{T-1}{M-1} .
\end{aligned}
$$

Since $\Gamma, T, M \in \mathbb{N}$, then

$$
\Gamma \leq T-1 \text { if } T<M,
$$

where the condition would be expected to hold in practice, since a large value of $M$ is desirable for high frequency accuracy and $T$ is kept small to minimize the quantization noise power.

Theorems FG1 and DK1 are quite similar, in that they show that the amplitude of $t$ imposes a fundamental constraint on

\footnotetext{
${ }^{3} \mathrm{An}$ example of this would be a hybrid quantizer consisting of the MASH 1-1-1 quantizer followed by the mapping $y \mapsto(y+3)^{\frac{1}{2}}$. The output of the MASH quantizer is spur-free in the linear case, so this hybrid quantizer would be immune to nonlinearities of the form $N: x \mapsto x^{2}$, but would not be spur-free in the linear case.
}

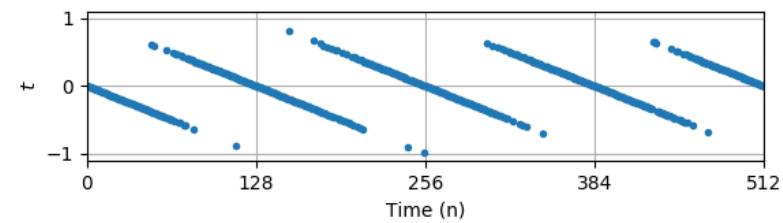

(a)

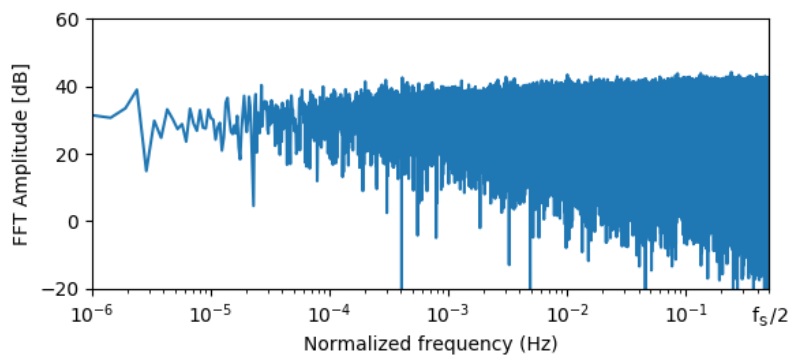

(b)

Fig. 5. Example of a quantizer exhibiting spur-free performance in the presence of a $\mathcal{N}: x \mapsto x^{8}$ nonlinearity, with a $T=2$. (a) $t$, and (b) the spectrum of $t_{\mathrm{nl}}$.

the number of nonlinearities for which spur-free operation can be guaranteed. The more general Theorem DK1 applies to any set of affine-unrelated nonlinearities, whereas Theorem FG1 is a special case that applies only to nonlinearities of the form $\mathcal{N}: x \mapsto x^{p}$ for values of $p \in[1,2, \ldots \Gamma]$.

From a practical perspective, Theorem FG1 describes constraints on obtaining immunity to spurs for power-of- $p$ nonlinearities for $p$ equal to or less than $\Gamma$, and finds that an amplitude of at least $T=\Gamma+1$ is required to achieve this. Theorem DK1 finds that, for any collection of $\Gamma$ non-trivial and affine-unrelated nonlinearities, an amplitude of at least $T=\Gamma+1$ is required to achieve this. The latter constraint is less restrictive in the sense that obtaining immunity to spurs for a known nonlinearity (in addition to the linear case) requires only an amplitude of $T=2$.

In the example of the $\Gamma^{\text {th }}$-order polynomial nonlinearity, knowledge of the polynomial coefficients allows one to reduce the required amplitude from $T=\Gamma+1$ to $T=2$. An example for the nonlinearity $\mathcal{N}: x \mapsto x^{8}$ is illustrated in Fig. 5: since the exact coefficients of the polynomial are known, $T=2$ is sufficient to satisfy the spur-free condition. For more complicated nonlinearities, spur-free operation is possible regardless of whether the nonlinearity can be expressed as a finite-order polynomial or not.

In addition to the above constraint, [24] also demonstrates the existence of an "optimal" quantizer for the class of polynomial nonlinearities of finite order, meaning that a quantizer can be described which would exhibit spur-free behavior corresponding to the limitation of Theorem FG1 for any order of polynomial. Theorem DK1 has loosened the constraints of Theorem FG1, so it is interesting to consider whether this new limit is actually achievable in general for an arbitrary collection of nonlinearities. We consider this question next.

The existence of an "optimal" quantizer for $p^{\text {th }}$-order polynomial nonlinearities can be worded as follows:

Theorem FG2: Consider a quantizer designed such that $t$ has 
an amplitude of $T$. For the set of nonlinear functions of the form $\mathcal{N}: x \mapsto x^{p}$, where $1 \leq p \leq T-1$, the quantizer can be designed such that $t_{\mathrm{nl}}$ is spur-free for each of these nonlinearities.

The corresponding statement in the context of an arbitrary collection of nonlinearities reads:

Theorem DK2: Consider a quantizer designed such that $t$ has an amplitude of $T$. There exists a set of $T-1$ or fewer arbitrary non-trivial and affine-unrelated nonlinear functions such that $t_{\mathrm{nl}}$ must contain spurs for at least one of these functions for any implementation of this quantizer.

It can be shown that an "optimal" quantizer does not exist in the case of an arbitrary collection of nonlinearities using a simple counterexample.

Proof. Consider, for the purposes of illustration, a quantizer designed such that $-2 \leq t \leq 2 \Longrightarrow T=4$. Consider two nonlinear functions: the unity function, $\mathcal{N}_{A}: x \mapsto x$, and the function $\mathcal{N}_{B}: x \mapsto x+\sin (2 \pi x) / 2$, both shown in Fig. 6(a).

The corresponding ranges of $t_{\mathrm{nl}}$, shown in Fig. 6(b), take the form $m-\langle n \alpha\rangle$ and $m-\langle n \alpha\rangle-\sin (2 \pi n \alpha)$, respectively. Since these ranges are linear with respect to $m$, the ensemble mean in each case evaluates to

$$
\begin{aligned}
\mu_{t_{\mathrm{nl}, \mathrm{A}}}[n]= & \left(\sum_{m} P_{t}(m-\langle n \alpha\rangle, n) m\right)-\langle n \alpha\rangle \\
\mu_{t_{\mathrm{n} l \mathrm{~B}}}[n]=\left(\sum_{m} P_{t}(m-\langle n \alpha\rangle, n) m\right) & -\langle n \alpha\rangle \\
& -\frac{1}{2} \sin (2 \pi n \alpha),
\end{aligned}
$$

where $t_{\mathrm{nl}, \mathrm{A}}, t_{\mathrm{nl}, \mathrm{A}}$ correspond to the application to $t$ of $\mathcal{N}_{A}$ and $\mathcal{N}_{B}$, respectively.

For spur-free operation in both cases, we require that $\mu_{t_{\mathrm{nl}, \mathrm{A}}}[n]=C_{A}$ and that $\mu_{t_{\mathrm{nl}, \mathrm{B}}}[n]=C_{B}$, where $C_{A}$ and $C_{B}$ are constants. Defining a third constant $C_{\Delta}=C_{A}-C_{B}$ and combining both equalities results in

$$
\begin{aligned}
C_{\Delta} & =\mu_{t_{\mathrm{nl}, \mathrm{A}}}[n]-\mu_{t_{\mathrm{nl}, \mathrm{B}}}[n] \\
& =\frac{1}{2} \sin (2 \pi n \alpha),
\end{aligned}
$$

where the right-hand side is independent of the choice of $T$ or $P_{t}(m-\langle n \alpha\rangle, n)$, and therefore applies to any quantizer.

Ignoring the trivial situation $\alpha=0$, this amounts to stating that the sine function evaluates to a constant for all values of the independent variable, which is a contradiction. Therefore, there exist pairings of nonlinear functions which are "incompatible" in the sense that ensuring spur-free operation for one guarantees the presence of spurs with the other.

In conclusion, [24] has defined the boundaries of achievable and unachievable performance for the quantizer in the presence of a nonlinearity described by a finite-order polynomial. Theorem DK1 has demonstrated that the boundary of unachievable performance can be reduced in the case of an arbitrary collection of nonlinearities, but it does not necessarily follow that the boundary of achievable performance can be similarly extended.

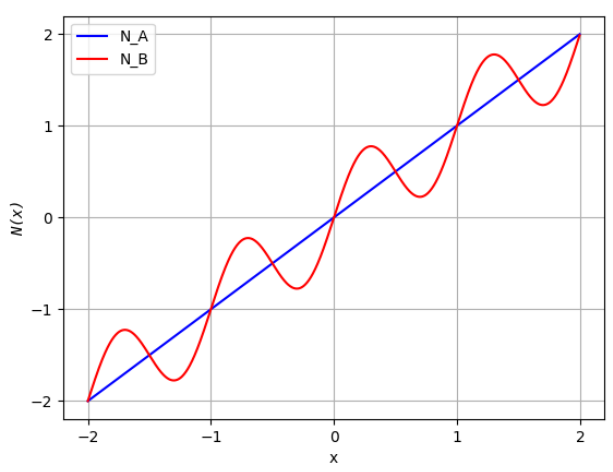

(a)

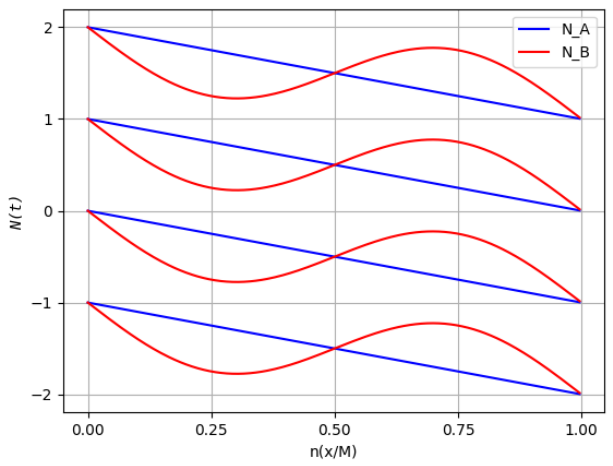

(b)

Fig. 6. Sample nonlinearities used in the indirect proof of Theorem DK2 (a) and corresponding range of $t_{\mathrm{nl}}(\mathrm{b})$.

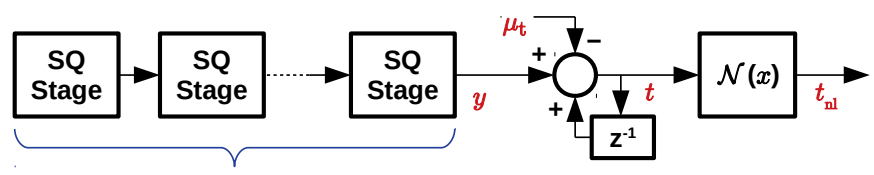

Fig. 7. Block diagram of quantizer and feedforward model employed in Section V-A. Not shown is the specification of the SQ stage transition matrices.

\section{EXAMPLES}

In this section, we will examine how the exposition presented above offers an intuitive explanation for the behavior of the Successive Requantizer, and demonstrate how this paper improves upon the constraints placed on spur-free operation with respect to [24].

\section{A. Describing the operation of an existing spur-free quantizer}

The class of quantizers called "Successive Requantizers," (SQ) offer spur-free operation in the presence of polynomial nonlinearities up to a specified order [9], [17], [18], [35]. The quantizer described in [17], for example, guarantees that $t$ does not produce spurs when it passes through polynomial nonlinearities of up to third order $\left(h_{t}=3\right.$ using that paper's terminology).

Fig. 7 shows a feedforward model of a first-order 8-stage SQ which implements the solution of [17]. Fig. 8 compares 


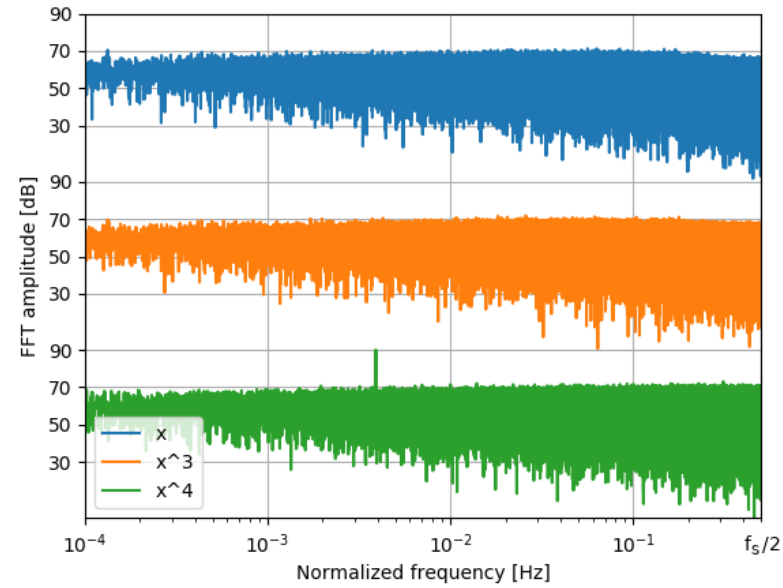

Fig. 8. Spectra of $t_{\mathrm{nl}}$ for a Successive Requantizer $\left(h_{t}=3\right)$, for the functions $\mathcal{N}_{1}: x \mapsto x$ (linear case), $\mathcal{N}_{3}: x \mapsto x^{3}$ and $\mathcal{N}_{4}: x \mapsto x^{4}$ (all spectra offset for clarity). A spur is present in the $\mathcal{N}_{4}$ case at $f=3.91 \times 10^{-3} \mathrm{~Hz}$, as this particular quantizer is only designed to offer spur-free operation for polynomial nonlinearities of order 3 or less.

the spectrum of $t_{\mathrm{nl}}$ for this quantizer $\left(X=1, M=2^{8}, n_{\mathrm{pts}}=\right.$ $2^{16}$ ), in the linear case (top), and after the application of two nonlinearities, $\mathcal{N}_{3}: x \mapsto x^{3}$ (middle) and $\mathcal{N}_{4}: x \mapsto x^{4}$ (bottom). As expected, the spectrum is spur-free in all but the $\mathcal{N}_{4}$ case, since this is a polynomial of order 4 .

In order to show how our analysis of spur production can explain the behavior of this quantizer with regard to fractional spurs, Eq. (11) was evaluated for each case. This required the determination of $P_{t}(x)$, which was determined theoretically as follows.

The Successive Requantizer consists of $K=8$ stages, whose operation is described by the transition matrices $A_{o}$ and $A_{e}$ associated with odd and even SQ stage outputs, respectively. In this case, the $A_{o}$ and $A_{e}$ matrices from [17] were employed to achieve spur-free operation for $\mathcal{N}_{3}$. It was assumed that the input to the $2^{\text {nd }}$ SQ stage onwards had a uniformly distributed parity, in which case the average of the even and odd transition matrices was taken to be the effective transition matrix:

$$
A=\frac{A_{o}+A_{e}}{2} \text {. }
$$

The PMF of the accumulated output of these stages is, therefore, given by the Steady-State Vector (SSV) of $A$, which in this case can be defined as the vector to which $P_{0}$.A.A..A converges for some valid $P_{0}$. For the state transition matrices employed above, it was calculated as $S S V=[1 / 16,1 / 4,3 / 8,1 / 4,1 / 16]$. The input to the first stage, however, is kept at a constant $X=1$ to produce a clear low-frequency fractional spur, hence the parity is always odd; therefore, for this stage the Steady-State Vector of $A_{o}$ was used instead, and was calculated as $S S V_{o}=$ $[3 / 40,1 / 5,9 / 20,1 / 5,3 / 40]$.

Since each stage is separated by a lossless 1-bit decimation (equivalent to a divide-by-2 operation), the contribution of successive stages was combined using the following iterative algorithm:

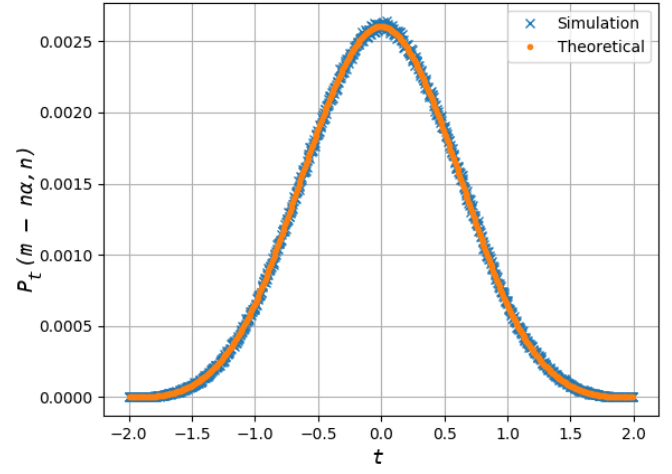

Fig. 9. Comparison of $P_{t}(x)$ derived from the Successive Requantizer's Transition Matrix and the histogram of the simulated $t$ signal.

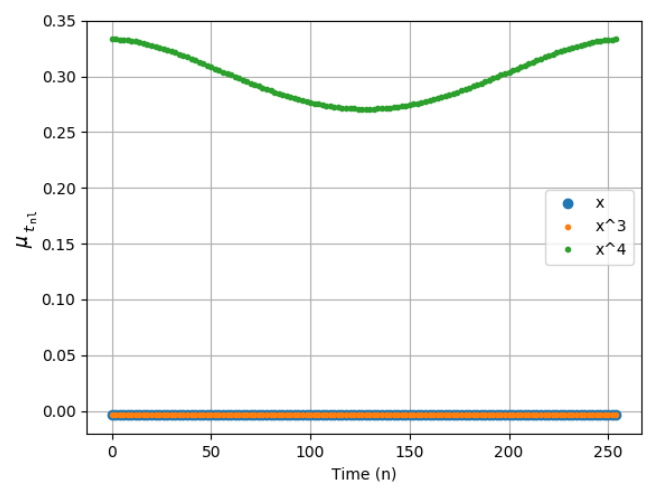

Fig. 10. Ensemble mean $\mu_{t_{\mathrm{nl}}}[n]$ after application of each of the nonlinearities shown in Fig. 8.

1) $P=S S V$ (contribution of stage $K$ ).

2) For each stage from $K-1$ to 2 inclusive:

a) Insert a 0 in between each element of $P$ to form $P^{\prime}$ (eg. $[1,2,3] \rightarrow[1,0,2,0,3]$ ).

b) $P=P^{\prime} * S S V$

3) For stage 1 only:

a) Insert a 0 in between each element of $P$ to form $P^{\prime}$ (eg. $[1,2,3] \rightarrow[1,0,2,0,3]$ ).

b) $P=P^{\prime} * S S V_{o}$

where $*$ denotes convolution. The resulting PMF is compared to the histogram of $t$ in Fig. 9, demonstrating that our theoretical derivation of $P_{t}(x)$ is accurate.

The value of the ensemble mean $\mu_{t_{\mathrm{nl}}}[n]$ from Eq. (11) using the derived $P_{t}(x)$ is illustrated over one $M$-length period in Fig. 10. For the $\mathcal{N}_{1}$ and $\mathcal{N}_{3}$ nonlinearity functions, $\mu_{t_{\mathrm{nl}}}[n]$ (bottom two traces) remains constant, indicating spur-free operation. In the $\mathcal{N}_{4}$ case, however, $\mu_{t_{\mathrm{nl}}}[n]$ (top trace) follows a sinusoid-like trajectory over time with a period of 256 , explaining the presence of a spur with a normalized frequency of $1 / 256=3.9 \times 10^{-3} \mathrm{~Hz}$, as seen in the bottom trace of Fig. 8. 


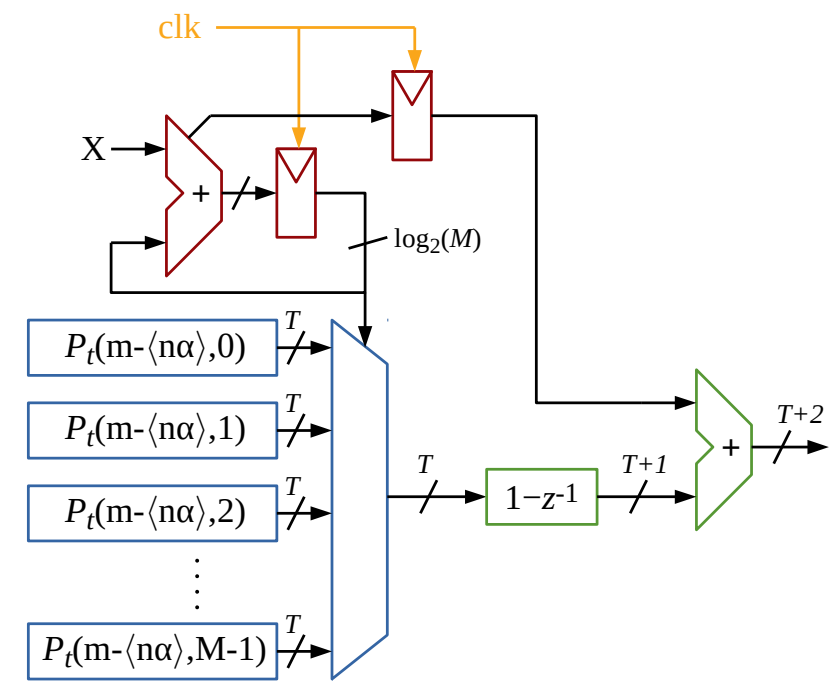

Fig. 11. Conceptual implementation of a quantizer with input $X$ implementing behaviour specified by an arbitrary $P_{t}(m-\langle n \alpha\rangle, n)$ probability matrix. $M$ random number generators, each exhibiting behavior corresponding to one of the $P_{t}(m-\langle n \alpha\rangle, n)$ rows, produce $M$ outputs, where $M$ is a power of 2 . These outputs are selected by a MUX indexed by a $\log _{2}(M)$-bit accumulator, whose overflow bit is added to the finite difference of the indexed random outputs to obtain the quantizer output. The output signal's mean is given by $\alpha=X / M$.

\section{B. A demonstration of optimum spur-free operation for an arbitrary, known nonlinearity}

The Successive Requantizer offers spur-free performance in the presence of an unknown polynomial nonlinearity of known order. Section IV has demonstrated that the amplitude of the quantizer output places a constraint on the number of qualitatively distinct nonlinearities for which spur-free operation can be guaranteed; therefore, the constraint of [24] is considerably loosened in the case of a known nonlinearity, meaning that spur-free operation can be guaranteed in principle for an arbitrary, but known quantizer with considerably less amplitude dynamic range than would be required for a similar spur-free Successive Requantizer.

Consider for example the $\mathcal{N}: x \mapsto x^{6}$ nonlinearity. A Successive Requantizer capable of guaranteeing spur-free operation for this nonlinearity, as well as the linear case $\mathrm{e}^{4}$, would necessarily produce a $t$ signal with an amplitude of at least 7 (in practice ${ }^{5}, 8$ ) in order to guarantee spur-free operation. Section IV demonstrates that the lower limit on the amplitude in this case is actually 3 (in practice ${ }^{5}, 4$ ), so the Successive Requantizer is sub-optimal when the nonlinearity is known explicitly.

Spur-free operation is guaranteed both before and after application of this nonlinearity if the quantizer is designed

\footnotetext{
${ }^{4}$ We expect the quantizer to demonstrate spur-free operation for both the nonlinear mapping function, $\mathcal{N}: x \mapsto x^{6}$, and the linear mapping function $\mathcal{L}: x \mapsto x$. Spur-free operation could be achieved with $T=2$ if an accurate description of the transfer function was available in place of an estimate of the nonlinearity, but the solution presented here is robust to scaling inaccuracies in the nonlinearity estimate.

${ }^{5}$ While it is entirely possible to implement a quantizer which produces an odd amplitude, for virtually all quantizers described in the literature the amplitude of $t$ is even because the output, $y$, is full-scale.
}

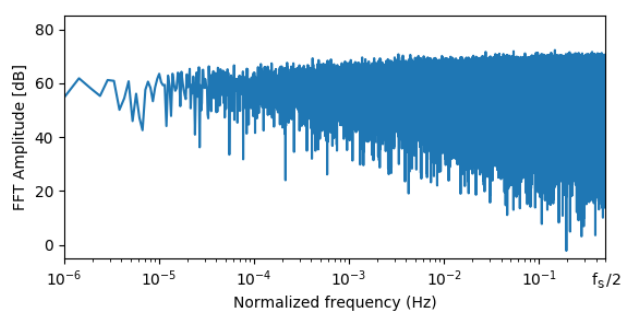

(a) $\mathcal{N}_{1}: x \mapsto x$

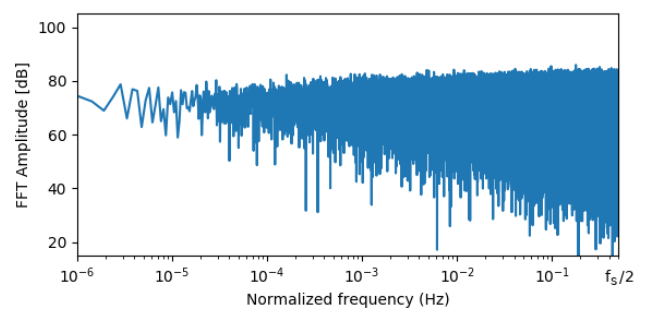

(b) $\mathcal{N}_{6}: x \mapsto x^{6}$

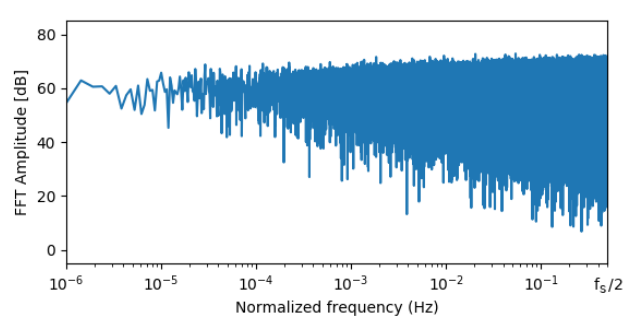

(c) $\mathcal{N}_{1,6}: x \mapsto x+0.1 x^{6}$

Fig. 12. Spectra of $t_{\mathrm{nl}}$ with designed quantizer for the linear (top) and $x^{6}$ (middle) mapping functions, as well as a combination of the two (bottom), showing spur-free behavior in all cases ( $2^{21}$ samples).

such that it implements $P_{t}(m-\langle n \alpha\rangle, n)$ values which produce a constant $\mu_{t}[n]$ and $\mu_{t_{\mathrm{nl}}}[n]$. One such matrix which satisfies this constraint for $M=2^{4}$ is:

$$
P_{t}(m-\langle n \alpha\rangle, n)=\left[\begin{array}{cccc}
0.3386 & 0.3281 & 0.3281 & 0.0052 \\
0.2981 & 0.3478 & 0.3478 & 0.0063 \\
0.2572 & 0.3678 & 0.3678 & 0.0072 \\
0.2162 & 0.3879 & 0.3879 & 0.0080 \\
0.1756 & 0.4077 & 0.4077 & 0.0090 \\
0.1362 & 0.4263 & 0.4263 & 0.0112 \\
0.0995 & 0.4421 & 0.4421 & 0.0163 \\
0.0679 & 0.4529 & 0.4529 & 0.0263 \\
0.0433 & 0.4567 & 0.4567 & 0.0433 \\
0.0262 & 0.4529 & 0.4529 & 0.0680 \\
0.0162 & 0.4421 & 0.4421 & 0.0996 \\
0.0112 & 0.4263 & 0.4263 & 0.1362 \\
0.0090 & 0.4077 & 0.4077 & 0.1756 \\
0.0079 & 0.3879 & 0.3879 & 0.2163 \\
0.0072 & 0.3678 & 0.3678 & 0.2572 \\
0.0064 & 0.3478 & 0.3478 & 0.2980
\end{array}\right] \text { (23) }
$$

for $-2<m \leq 2$ (ie. $T=4$ ) and $0 \leq n<2^{4}=M$.

It can be verified directly from the above matrix that

$$
\mu_{t}[n]=\sum_{m} P_{t}(m-\langle n \alpha\rangle, n)(m-\langle n \alpha\rangle)=0 \quad \forall n,
$$


and

$$
\mu_{t_{\mathrm{nl}}}[n]=\sum_{m} P_{t}(m-\langle n \alpha\rangle, n)(m-\langle n \alpha\rangle)^{6}=1 \quad \forall n,
$$

thus satisfying the spur-free conditions - that $\mu_{t}[n]$ and $\mu_{t_{\mathrm{nl}}}[n]$ are constant over $n$-for any quantizer input value $X$. Note that the solution given by Eq. (23) satisfies $P(0-\langle n \alpha\rangle, n)=$ $P(1-\langle n \alpha\rangle, n) \forall n$; the problem is underconstrained when the amplitude is greater than 3 .

Fig. 11 shows a conceptual implementation of a quantizer exhibiting the behavior defined by Eq. (23), where each of the rows in the matrix corresponds to a separate random number generator. Fig. 12 shows the accumulated output spectrum of this quantizer after application of the linear and $x^{6}$ nonlinear mapping functions, confirming spur-free behaviour. A spurfree spectrum is also obtained after application of a linear combination of the two mapping functions, showing that the solution is robust in the presence of gain and offset errors.

\section{CONCLUSIONS}

In this paper, we have offered a different perspective on the results presented in [24], extending the analysis to nonlinearities other than polynomials.

We have presented a framework for gaining insight into spur generation that arises when a quantizer's accumulated output encounters a memoryless nonlinearity. We have also shown that the ensemble mean of the accumulated zero-mean quantizer output is directly responsible for the presence or absence of these spurs.

We have determined an upper bound on the number of distinct nonlinear functions for which spur-free operation can be guaranteed using a single quantizer design, and have shown that this bound is reduced if the nonlinear functions place conflicting requirements on the statistics of the quantizer. We have also shown that it is possible in principle to design a quantizer with guaranteed spur-free performance for any arbitrary but known nonlinearity function.

\section{REFERENCES}

[1] S. Pamarti and I. Galton, "LSB Dithering in MASH DeltaSigma D/A Converters," IEEE Transactions on Circuits and Systems I: Regular Papers, vol. 54, no. 4, pp. 779-790, April 2007.

[2] B. Miller and B. Conley, "A multiple modulator fractional divider," in 44th Annual Symposium on Frequency Control, May 1990, pp. 559-568.

[3] B. De Muer and M. Steyaert, "A CMOS monolithic $\Delta \Sigma$-controlled fractional- $N$ frequency synthesizer for DCS-1800," Solid-State Circuits, IEEE Journal of, vol. 37, no. 7, pp. 835-844, Jul 2002.

[4] H. Hedayati, B. Bakkaloglu, and W. Khalil, "Closed-Loop Nonlinear Modeling of Wideband $\Sigma \Delta$ Fractional- $N$ Frequency Synthesizers," IEEE Transactions on Microwave Theory and Techniques, vol. 54, no. 10, pp. 3654-3663, Oct 2006.

[5] M. P. Kennedy, "Recent advances in the analysis, design and optimization of digital delta-sigma modulators," Nonlinear Theory and Its Applications, IEICE, vol. 3, no. 3, pp. 258-286, 2012.

[6] S. E. Meninger and M. H. Perrott, "A 1-MHZ bandwidth 3.6-GHz $0.18-\mu \mathrm{m}$ CMOS fractional- $N$ synthesizer utilizing a hybrid PFD/DAC structure for reduced broadband phase noise," IEEE Journal of SolidState Circuits, vol. 41, no. 4, pp. 966-980, April 2006.

[7] T. A. D. Riley, N. M. Filiol, Qinghong Du, and J. Kostamovaara, "Techniques for in-band phase noise reduction in $\Delta \Sigma$ synthesizers," IEEE Transactions on Circuits and Systems II: Analog and Digital Signal Processing, vol. 50, no. 11, pp. 794-803, Nov 2003.
[8] W. Rhee and A. Ali, "An on-chip phase compensation technique in fractional- $N$ frequency synthesis," in Circuits and Systems, 1999. ISCAS '99. Proceedings of the 1999 IEEE International Symposium on, vol. 3, Jul 1999, pp. 363-366 vol.3.

[9] A. Swaminathan, A. Panigada, E. Masry, and I. Galton, "A digital requantizer with shaped requantization noise that remains well-behaved after nonlinear distortion," IEEE Trans. Signal Process., vol. 55, no. 11, pp. 5382-5394, November 2007.

[10] L. Zhang, X. Yu, Y. Sun, W. Rhee, Z. Wang, H. Chen, and D. Wang, "A hybrid spur compensation technique for finite-modulo fractional- $N$ phase-locked loops," in 2008 IEEE Asian Solid-State Circuits Conference, Nov 2008, pp. 417-420.

[11] L. Zhang, X. Yu, Y. Sun, W. Rhee, D. Wang, Z. Wang, and H. Chen, "A hybrid spur compensation technique for finite-modulo fractional$N$ phase-locked loops," IEEE Journal of Solid-State Circuits, vol. 44, no. 11, pp. 2922-2934, Nov 2009.

[12] H. Arora, N. Klemmer, J. C. Morizio, and P. D. Wolf, "Enhanced Phase Noise Modeling of Fractional-N Frequency Synthesizers," IEEE Transactions on Circuits and Systems I: Regular Papers, vol. 52, no. 2, pp. 379-395, Feb 2005.

[13] P. V. Brennan, H. Wang, D. Jiang, and P. M. Radmore, "A new mechanism producing discrete spurious components in fractional- $n$ frequency synthesizers," IEEE Transactions on Circuits and Systems I: Regular Papers, vol. 55, no. 5, pp. 1279-1288, June 2008.

[14] S. Levantino, L. Collamati, C. Samori, and A. L. Lacaita, "Folding of Phase Noise Spectra in Charge-Pump Phase-Locked Loops Induced by Frequency Division," IEEE Transactions on Circuits and Systems II: Express Briefs, vol. 57, no. 9, pp. 671-675, Sep 2010.

[15] B. Razavi, "An alternative analysis of noise folding in fractional-n synthesizers," in 2018 IEEE International Symposium on Circuits and Systems (ISCAS), May 2018, pp. 1-4.

[16] Y. Donnelly and M. P. Kennedy, "Prediction of phase noise and spurs in a nonlinear fractional- $N$ frequency synthesizer," IEEE Transactions on Circuits and Systems I: Regular Papers, vol. 66, no. 11, pp. 4108-4121, Nov 2019.

[17] E. Familier, C. Venerus, and I. Galton, "A class of quantizers with DCfree quantization noise and optimal immunity to nonlinearity-induced spurious tones," IEEE Transactions on Signal Processing, vol. 61, no. 17, pp. 4270-4283, Sept 2013.

[18] E. Familier and I. Galton, "Second and Third-Order Noise Shaping Digital Quantizers for Low Phase Noise and Nonlinearity-Induced Spurious Tones in Fractional- $N$ PLLs," IEEE Transactions on Circuits and Systems I: Regular Papers, vol. 63, no. 6, pp. 836-847, June 2016.

[19] K. J. Wang, A. Swaminathan, and I. Galton, "Spurious -tone suppression techniques applied to a wide-bandwidth 2.4ghz fractional-n pll," in 2008 IEEE International Solid-State Circuits Conference - Digest of Technical Papers, 2008, pp. 342-618.

[20] E. Familier and I. Galton, "Second and third-order successive requantizers for spurious tone reduction in low-noise fractional-n plls," in 2017 IEEE Custom Integrated Circuits Conference (CICC), 2017, pp. 1-4.

[21] M. Perrott, M. Trott, and C. Sodini, "A modeling approach for $\Sigma$ $\Delta$ fractional- $N$ frequency synthesizers allowing straightforward noise analysis," Solid-State Circuits, IEEE Journal of, vol. 37, no. 8, pp. 1028 1038, Aug 2002.

[22] B. De Muer and M. S. J. Steyaert, "On the analysis of /spl delta//spl sigma/ fractional-n frequency synthesizers for high-spectral purity," IEEE Transactions on Circuits and Systems II: Analog and Digital Signal Processing, vol. 50, no. 11, pp. 784-793, 2003.

[23] K. Hosseini, B. Fitzgibbon, and M. Kennedy, "Observations concerning the generation of spurious tones in digital delta-sigma modulators followed by a memoryless nonlinearity," Circuits and Systems II: Express Briefs, IEEE Transactions on, vol. 58, no. 11, pp. 714-718, Nov 2011.

[24] E. Familier and I. Galton, "A fundamental limitation of DC-free quantization noise with respect to nonlinearity-induced spurious tones," IEEE Transactions on Signal Processing, vol. 61, no. 16, pp. 4172-4180, Aug 2013.

[25] D. Bannerjee, PLL Performance, Simulation and Design. Dog Ear Publishing, 2006

[26] C. Weltin-Wu, E. Temporiti, M. Cusmai, D. Baldi, and F. Svelto, "Insights into wideband fractional adplls: Modeling and calibration of nonlinearity induced fractional spurs," IEEE Transactions on Circuits and Systems I: Regular Papers, vol. 57, no. 9, pp. 2259-2268, 2010.

[27] D. Biswas and T. Kanti Bhattacharyya, "A model of spurs for deltasigma fractional plls," in 2019 32nd International Conference on VLSI Design and 2019 18th International Conference on Embedded Systems (VLSID), 2019, pp. 512-513. 
[28] K. Stowe, An Introduction to Thermodynamics and Statistical Mechanics, Second Edition. Cambridge University Press, 2007.

[29] D. A. McQuarrie, Statistical Thermodynamics. Harper \& Row, 1973.

[30] E. Temporiti, G. Albasini, I. Bietti, R. Castello, and M. Colombo, "A 700-kHz bandwidth $\Sigma \Delta$ fractional synthesizer with spurs compensation and linearization techniques for WCDMA applications," IEEE Journal of Solid-State Circuits, vol. 39, no. 9, pp. 1446-1454, Sept 2004.

[31] M. Kozak and I. Kale, "Rigorous analysis of delta-sigma modulators for fractional-n pll frequency synthesis," IEEE Transactions on Circuits and Systems I: Regular Papers, vol. 51, no. 6, pp. 1148-1162, June 2004.

[32] K. Hosseini and M. P. Kennedy, Minimizing Spurious Tones in Digital Delta-Sigma Modulators. Springer Science \& Business Media, 2011.

[33] V. R. Gonzalez-Diaz, M. A. Garcia-Andrade, G. E. Flores-Verdad, and F. Maloberti, "Efficient Dithering in MASH Sigma-Delta Modulators for Fractional Frequency Synthesizers," IEEE Transactions on Circuits and Systems I: Regular Papers, vol. 57, no. 9, pp. 2394-2403, Sept 2010.

[34] H. Mo and M. P. Kennedy, "Masked dithering of MASH digital deltasigma modulators with constant inputs using multiple linear feedback shift registers," IEEE Transactions on Circuits and Systems I: Regular Papers, vol. 64, no. 6, pp. 1390-1399, June 2017.

[35] K. J. Wang, A. Swaminathan, and I. Galton, "Spurious Tone Suppression Techniques Applied to a Wide-Bandwidth 2.4 GHz Fractional-N PLL," IEEE Journal of Solid-State Circuits, vol. 43, no. 12, pp. 2787-2797, Dec 2008 